\title{
The Preparation of $\alpha$-Haloalkyl Esters
}

\author{
ERKKI K. EURANTO, ASKO NOPONEN and TAUNO KUJANPÄ̈̈
}

Department of Chemistry, University of Turku, Turku, Finland

\begin{abstract}
The preparation and physical constants of 18 new or imperfectly known $\alpha$-haloalkyl esters of aliphatic acids are reported. The compounds include four monochloromethyl esters, seven other $\alpha$-monochloroalkyl esters, bromomethyl chloroacetate, iodomethyl and $\alpha$ iodoethyl acetates, three dichloromethyl esters, and trichloromethyl trichloroacetate. The esters were prepared by known methods with improvements especially in the purification stage, including selective hydrolysis of the products. A critical survey of the synthetic methods is given. A few other compounds, including two new methylene bischloropropionates, were obtained as by-products.
\end{abstract}

The methods for the preparation and purification of $\alpha$-monohaloalkyl 1 esters have been previously reviewed and discussed by the authors. ${ }^{1-3}$ Also the preparation of several individual esters was described in connection with kinetic studies. ${ }^{4-9}$ The main purpose of this paper is to report preparative methods and physical data for several new or imperfectly characterised $\alpha$-haloalkyl esters, including di- and trichloromethyl esters, that were needed in reaction kinetic studies. Preparative methods for $\alpha$-haloalkyl esters are reviewed in general, and improvements in the purification procedures are suggested.

1. The chlorination of alkyl esters can be performed either by chlorine, preferably in ultraviolet light, or by sulphuryl chloride (for references, see Ref. 1). The first method seems to be superior to the latter. ${ }^{10}$ Chlorination methods are appropriate only when other methods cannot be used (e.g., for chloromethyl formate ${ }^{1}$ and many $\alpha$-polychloroalkyl esters) or give poor yields (e.g., $\alpha$-chloroalkyl haloacetates ${ }^{4,7}$ ); the opposite view, however, has also been presented. 11,12 Chlorination cannot be used in the preparation of aliphatic $\alpha$-chloroalkyl esters having four or more carbon atoms in the alkyl or acyl component of the ester, ${ }^{13,14}$ except when all other hydrogen atoms are or will be substituted by halogens. ${ }^{15}$

The most serious disadvantage of the chlorination method is that in addition to the desired compound also isomeric and polysubstituted esters are formed, ${ }^{1}$ and these are often difficult to remove by distillation because of close boiling points (see, e.g., the data for the three chloromethyl trichloroacetates

Acta Chem. Scand. 20 (1966) No. 5 


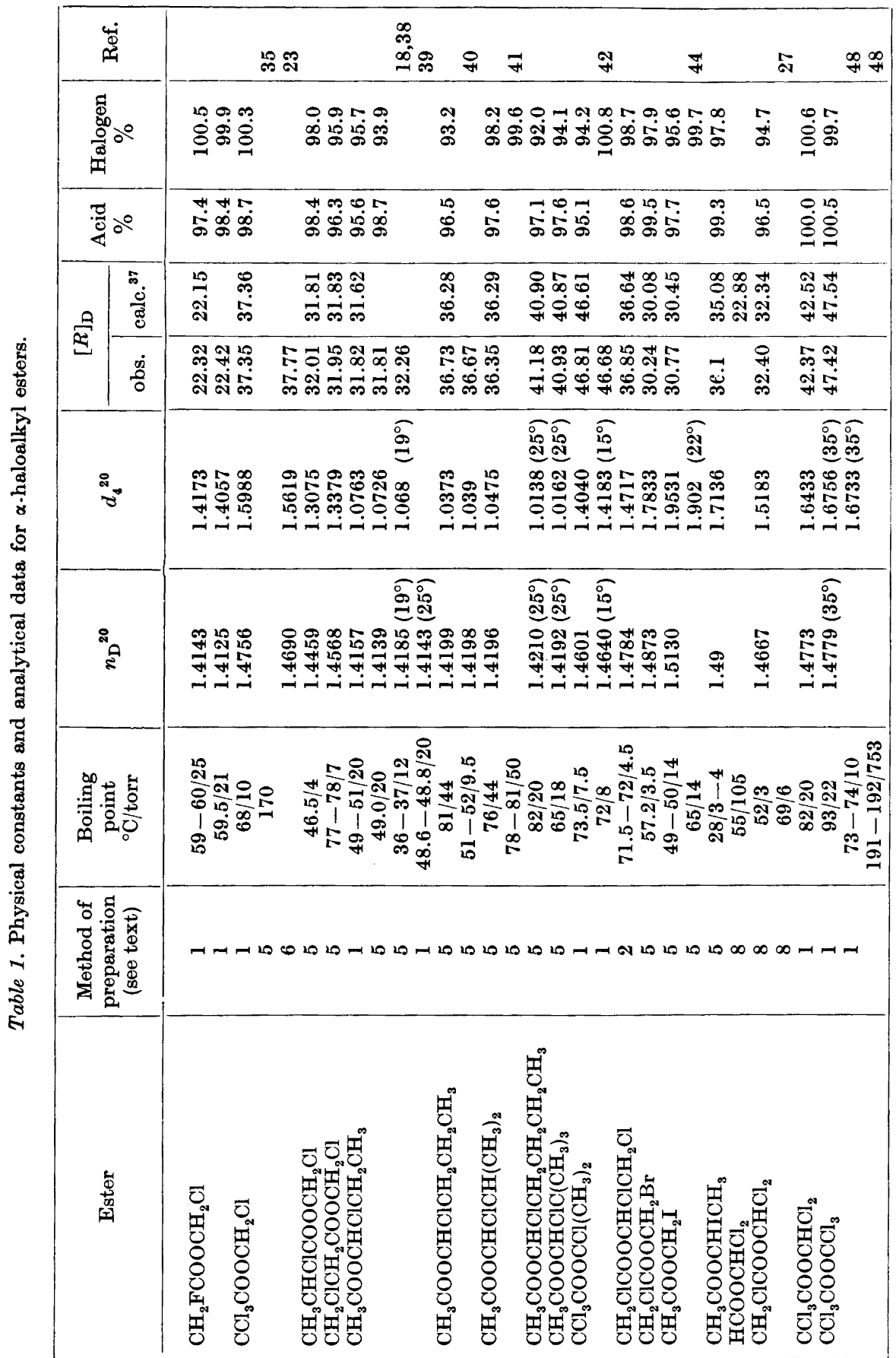

Acta Chem. Scand. 20 (1966) No. 5 
in Table 1). When chloromethyl esters were prepared, the difficulty was avoided by the following procedure. When a monochloromethyl ester is desired, the chlorination is discontinued at an early stage when only slight polychlorination has occurred. The product can then be isolated by distillation from the unreacted and acyl-substituted esters because of the relatively large boiling point differences. In some cases, however, significant formation of the dichloromethyl ester cannot be avoided. The latter ester is then removed by the selective hydrolysis method described below. When a dichloromethyl ester is the desired product, the chlorination is continued until the monosubstituted product has reacted. After the acyl-substituted esters and other removable impurities are distilled off, the ester with an excess of alkyl chlorine atoms is hydrolysed by a calculated amount of water dissolved in acetone. This method is based on the fact, found in kinetic measurements, that every additional chlorine atom in the methyl group of the alkyl component increases the rate of the neutral hydrolysis of the ester by a factor varying from about eleven to several hundreds, so that only a small calculable amount of the desired ester is destroyed. By this method, dichloromethyl trichloroacetate was obtained in high purity (Table 1).

2. The addition of halogen to the double bond of $\alpha$-alkenyl esters ${ }^{16}$ is evidently the best method for the preparation of primary $\alpha, \beta$-dihaloalkyl esters, because no impurities are formed that are difficult to remove. The reaction cannot be used to prepare secondary $\alpha, \beta$-dihaloalkyl esters because the formed esters decompose immediately to acyl halides and $\alpha$-haloketones.

3. The addition of hydrogen halide to $\alpha$-alkenyl esters ${ }^{17}$ or to allylidene diacetate ${ }^{18}$ leads to $\alpha$-haloalkyl esters or $\alpha, \gamma$-dihalopropyl acetate, respectively. The value of these preparative reactions is limited, because the esters in question can usually be prepared more conveniently by other methods.

4. The addition of tetrahalomethanes to vinyl acetate in the presence of acetyl peroxide gives $\alpha, \gamma, \gamma, \gamma$-tetrahalopropyl acetates. ${ }^{19}$

5. The reaction between an acyl halide and an aldehyde (for references, see Ref. 1) or a ketone ${ }^{2,3}$ is without doubt the most useful method for the preparation of $\alpha$-monohaloalkyl esters of most acids (see, however, section 1). The reaction of an acyl halide with higher aldehydes, including propionaldehyde, ${ }^{18}$ and the reaction of acetyl iodide with aldehydes (see Experimental Part) take place easily without catalyst, but in the case of reactions between other acyl halides and aldehydes it is advantageous to use anhydrous zinc chloride as catalyst, ${ }^{20}$ whereas the reactions between acyl halides and most ketones require the use of anhydrous aluminium chloride or boron trifluoride as catalyst. ${ }^{3,21}$ It is advisable to avoid unnecessary heating during the reaction and distillation while the catalyst is still present, because it may lead to undesired side reactions and decomposition of the ester. Impurities like $\alpha, \alpha^{\prime}$-dihaloalkyl ethers and alkylidene diesters ${ }^{22}$ can, however, be removed by careful distillation in most cases. ${ }^{1,12}$ The alkylidene diester may be the main product of the reaction between an acyl halide with electronegative substituents and an aldehyde (see section 1 and Experimental Part), whereas no reaction was found to take place between acetyl chloride and ketones with bulky groups even in the presence of anhydrous aluminium chloride (see Experimental Part).

Acta Chem. Scand. 20 (1966) No. 5 
6. The reactions of carboxylic acids with paraformaldehyde and gaseous hydrogen chloride in the presence of anhydrous zinc chloride at -15 to $0^{\circ} \mathrm{C}$ has been stated by Pishnamazzade et al..$^{23}$ to lead to chloromethyl esters. However, repeated attempts to prepare chloromethyl caproate and trichloroacetate by this method did not give isolatable amounts of the esters. On the other hand, the physical data given by Pishnamazzade et al. ${ }^{23}$ differ significantly from those found for the compounds in question that have been prepared by other methods.* This method seems therefore to be less advantageous.

7. Acyl halides are reported to produce $\alpha$-haloalkyl esters also when they react with 1,3-dioxanes containing an alkyl group vicinal to one of the ring oxygen atoms, ${ }^{24}$ with 2,3-dichlorotetrahydrofuran ${ }^{25}$ and with epoxides. ${ }^{26}$

8. The reaction of phenyl(bromodichloromethyl)mercury with carboxylic acids in benzene solution at $60-80^{\circ} \mathrm{C}$ gives dichloromethyl esters ${ }^{27}$ and seems to be the most general preparative method for these esters and is of especial value when direct chlorination cannot be used. It was found that better yields of phenyl(bromodichloromethyl)mercury were obtained from phenylmercuric bromide or chloride, bromodichloromethane, and potassium tert-butoxide when diethyl ether was used as solvent at about $-20^{\circ} \mathrm{C}$ instead of benzene at $0^{\circ} \mathrm{C}$ as proposed originally. ${ }^{28}$

9. Electrolysis of carboxylic acids and their alkali metal salts with one, two, or three halogen atoms in the $\alpha$-position gives $\alpha$-haloalkyl esters of $\alpha$-haloacids. ${ }^{29,30}$ This seems to be the most convenient method for the preparation of several esters with two or three halogen atoms in the $\alpha$-positions in the acyl and alkyl components..$^{30,31}$

10. The reaction between mercuric difluoroacetate and iodine or difluoroiodomethane has been reported to give difluoromethyl difluoroacetate. ${ }^{32}$ Difluoromethyl trifluoroacetate has been obtained as a decomposition product of perfluoro-3,5-dimethyl-1,2-dioxolane, probably by a reaction of trifluoroacetic acid with difluoromethylene ${ }^{33}$ (cf. method 8 ), which reaction has been found to take place. ${ }^{34}$

\section{EXPERIMENTAL}

The starting materials and the preparative method for each ester are given in the following. The procedures employed have been described in greater detail earlier. ${ }^{1-9}$ The chlorinations were performed with gaseous chlorine in ultraviolet light employing a Hanovia 11 Photochemical Reactor.

The physical constants of the esters are given together with earlier data, when available, in Table 1. The infrared spectra of the chloroderivatives of methyl acetate have been reported and discussed by Laato. ${ }^{31}$ The esters were analysed by hydrolysing weighed amounts in water, or in a known excess of dilute sodium hydroxide solution, if the neutral solvolysis was too slow. The solutions were titrated with standard sodium hydroxide or hydrochloric acid and silver nitrate solutions. The results are given in Table 1 in the columns headed "Acid \%" and "Halogen \%" as percentages of the theoretical amounts taking into account that one mole of an $\alpha$-monohaloalkyl ester gives on hydrolysis two moles of acid and one mole of halide ion, whereas the corresponding numbers are 3 and 2 for dichloromethyl esters and 4 and 3 for trichloromethyl esters.

\footnotetext{
* Pishnamazzade et al. ${ }^{23}$ do not give boiling points. For the refractive index and density of chloromethyl propionate they give the values $n_{\mathrm{D}}{ }^{20}=1.4295$ and $d_{4}^{20}=1.1824$, whereas we have found ${ }^{x}$ the values 1.4150 and 1.1364 , respectively; for data for chloromethyl trichloroacetate, see Table 1 .
} 
Paraformaldehyde (E. Merck AG) and anhydrous zinc chloride (May \& Baker Ltd.) were used as received. Methanol (E. Merck AG, Guaranteed Reagent) was dried with magnesium methoxide. Acetyl chloride (May \& Baker Ltd., or E. Merck AG) was distilled before use. Fluoroacetyl chloride (b.p. $72^{\circ} \mathrm{C}$ ) was prepared by dropping melted fluoroacetic acid (Fluka AG, purum) slowly onto phosphorus pentachloride (May \& Baker Ltd.). Chloroacetyl bromide (b. p. $127^{\circ} \mathrm{C}$ ) was prepared from bromine (E. Merck AG), red phosphorus (British Drug Houses Ltd.), and chloroacetic acid (E. Merck AG). Acetyl iodide was prepared from iodine (E. Merck AG, resublimed), acetic anhydride (E. Merck AG, pure), and red phosphorus (British Drug Houses Ltd.). All of the acyl halides were distilled twice before use in the syntheses.

Methyl fluoroacetate (b.p. $104^{\circ} \mathrm{C}$ ) was prepared from methanol and fluoroacetyl chloride, methyl trichloroacetate $\left(n_{\mathrm{D}}{ }^{20} 1.4570, d_{4}{ }^{20} 1.4855\right)$ from trichloroacetic acid (E. Merck AG, Guaranteed Reagent) and methanol, and propyl acetate (b.p. $102^{\circ} \mathrm{C} / 766$ torr, $n_{\mathrm{D}}{ }^{20} 1.3838$ ) from acetic acid (E. Merck $A G$ ) and propyl alcohol (E. Merck $A G$ ) in the presence of concentrated sulphuric acid. Vinyl chloroacetate (b.p. $37-38^{\circ} \mathrm{C} / 16$ torr, $n_{\mathrm{D}}{ }^{25} 1.4422$ ) was prepared from chloroacetic acid and acetylene with yellow mercuric oxide as catalyst.

Valeraldehyde (b.p. $104^{\circ} \mathrm{C} / 755$ torr, m.p. of the 2,4 -dinitrophenylhydrazone $99^{\circ} \mathrm{C}$ ) was prepared by oxidation of pentyl alcohol (British Drug Houses Ltd.) with chromic acid (from sodium dichromate and sulphuric acid). The yield was low. The distillation residue contained appreciable amounts of pentyl valerate (b.p. $200-205^{\circ} \mathrm{C} / 757$ torr). Pivalaldehyde (2,2-dimethylpropanal, b.p. $74^{\circ} \mathrm{C} / 755$ torr $-77^{\circ} \mathrm{C} / 761$ torr, $n_{\mathrm{D}}{ }^{25} 1.3792$ ) was prepared by the following reaction sequence: tert-Butyl chloride (b.p. $50.3^{\circ} \mathrm{C} / 755$ torr), prepared from tert-butyl alcohol (British Drug Houses Ltd.) and concentrated hydrochloric acid, was transformed into neopentyl alcohol (b.p. $110-112^{\circ} \mathrm{C} / 751$ torr) by a Grignard reaction. The alcohol was oxidised to pivalaldehyde with chromic acid or with tert-butyl chromate. With the first oxidant, neopentyl pivalate (b.p. $164^{\circ} \mathrm{C} / 750$ torr) was obtained as by-product.

Phenyl(bromodichloromethyl)mercury ${ }^{28}$ was prepared from phenylmercuric chloride (British Drug Houses Ltd.) or bromide (recovered from the reaction mixtures after the preparation and isolation of dichloromethyl esters), bromodichloromethane (prepared from chloroform and ethyl bromide with aluminium chloride as catalyst), and potassium tert-butoxide (from potassium metal (Coleman \& Bell Co.) and tert-butyl alcohol) in diethyl ether solution at about $-20^{\circ} \mathrm{C}$.

Chloromethyl fluoroacetate was prepared by chlorinating methyl fluoroacetate (method 1). It was not obtained from fluoroacetyl chloride and paraformaldehyde (method 5).

Chloromethyl trichloroacetate ${ }^{35,36,23}$ was prepared by chlorinating methyl trichloroacetate (method 1). The distilled ester was found to contain a significant amount of dichloromethyl trichloroacetate,$^{36}$ which was removed by hydrolysis with a calculated volume of $3 \%$ water-acetone and redistillation. An attempt was made to synthesize the ester also from trichloroacetic acid, paraformaldehyde, and hydrogen chloride gas in the presence of zinc chloride (method 6). ${ }^{23}$ A liquid reaction product was obtained, but no chloromethyl trichloroacetate could be isolated.

Chloromethyl $\alpha$-chloropropionate was prepared from $\alpha$-chloropropionyl chloride (Eastman Organic Chemicals) and paraformaldehyde in the presence of zinc chloride (method 5). The mixed reagents were heated at $60^{\circ} \mathrm{C}$ for $18 \mathrm{~h}$. Yield $25 \%$. A higher boiling fraction $(50 \%)$ was found to be methylene bis- $\alpha$-chloropropionate (b.p. $158^{\circ} \mathrm{C} / 11$ torr, $n_{\mathrm{D}}{ }^{20} 1.4559, d_{4}{ }^{20} 1.3111,[R]_{\mathrm{D}} 47.48$, calc. ${ }^{37} 47.28$ ). Analytical data: $0.6086 \mathrm{mmole}$ of the latter ester gave on hydrolysis 1.102 mmoles of acids $(90.5 \%)$ and 0.0074 mmole of chloride.

Chloromethyl $\beta$-chloropropionate was prepared from $\beta$-chloropropionyl chloride (Eastman Organic Chemicals) and paraformaldehyde in the presence of zinc chloride (method 5) at room temperature. Yield $29 \%$. A higher-boiling fraction (22\%) was found to be methylene bis- $\beta$-chloropropionate (b.p. $160^{\circ} \mathrm{C} / 7$ torr, $n_{\mathrm{D}}{ }^{20} 1.4647, d_{4}{ }^{20} 1.3337,[R]_{\mathrm{D}}$ 47.45, calc $^{32} 47.32$ ). Analytical data: 0.3846 mmole of the latter ester gave on total hydrolysis (by refluxing $80 \mathrm{~min}$ with about $2 \mathrm{mmoles}$ of sodium hydroxide in water) $1.542 \mathrm{mmoles}$ of acids (100.2\%) and 0.7652 mmole of chloride $(99.5 \%)$.

$\alpha$-Chloropropyl acetate ${ }^{18,38,39}$ was prepared by adding propionaldehyde (Judex Chemicals) slowly to cooled acetyl chloride to which zinc chloride had been added (method 5). The ester was also obtained by chlorinating cold propyl acetate with chlorine gas in

Acta Chem. Scand. 20 (1966) No. 5 
ultraviolet light (method 1). The monochloropropyl acetates (42\%) were fractionated by careful distillation with the following yields: $18 \mathrm{~g}$ of $\alpha$-chloropropyl acetate (Table 1), $36 \mathrm{~g}$ of $\beta$-chloropropyl acetate (b.p. $153^{\circ} \mathrm{C} / 771$ torr, $n_{\mathrm{D}}{ }^{20} 1.4206$ ), and $33 \mathrm{~g}$ of $\gamma$-chloropropyl acetate (b.p. $168^{\circ} \mathrm{C} / 771$ torr, $\left.n_{\mathrm{D}}{ }^{20} 1.4288\right)$. The isomer distribution $(21: 41: 38)$ is similar to that obtained by Brown and Ash ${ }^{39}$ after photochlorination in carbon tetrachloride solution at $80-85^{\circ} \mathrm{C}(23: 42: 35)$ and after chlorination with sulphuryl chloride (25:46:29). $\alpha$-Chlorobutyl acetate ${ }^{40}$ was prepared from acetyl chloride and freshly distilled butyraldehyde (L. Light \& Co., Ltd., b.p. $75^{\circ} \mathrm{C} / 763$ torr) in the presence of zinc chloride (method 5).

$\alpha$-Chloroisobutyl acetate ${ }^{41}$ was prepared from acetyl chloride and isobutyraldehyde (L. Light \& Co., Ltd.) in the presence of zinc chloride (method 5). Yield ca. $35 \%$.

$\alpha$-Chloropentyl acetate was prepared from acetyl chloride and valeraldehyde without catalyst at room temperature (method 5). The ester was not obtained in a high degree of purity because only a couple of grams of the aldehyde was available.

$\alpha$-Chloroneopentyl acetate was prepared from acetyl chloride and pivalaldehyde without catalyst (method 5). The reaction mixture was cooled at the beginning of the reaction and warmed slightly before distillation.

$\alpha$-Chloroisopropyl trichloroacetate ${ }^{42}$ was prepared by chlorinating isopropyl acetate ${ }^{43}$ (method 1). Yield $21 \%$.

Attempts to prepare the acetates of 2-chloro-3,3-dimethyl-2-butanol and 3-chloro2,2,4,4-tetramethyl-3-pentanol from acetyl chloride and the corresponding ketones with aluminium chloride as catalyst (method 5$)^{3}$ failed because no reaction took place, possibly because of steric hindrance. An attempt was also made to prepare the first-mentioned ester from the acetate of 3,3-dimethyl-1-buten-2-ol and hydrogen chloride (method 3), but the ester, probably formed as an intermediate, decomposed into acetyl chloride and pinacolone (methyl tert-butyl ketone); cf. Ref. 2.

$\alpha, \beta$-Dichloroethyl chloroacetate ${ }^{16}$ was prepared by adding chlorine to vinyl chloroacetate in the dark at $0^{\circ} \mathrm{C}$ (method 2$)$. Yield $45 \%$.

Bromomethyl chloroacetate was prepared from chloroacetyl bromide and paraformaldehyde with zinc chloride as catalyst (method 5). Yield $10 \%$.

Iodomethyl acetate 44 was prepared from acetyl iodide and paraformaldehyde without catalyst (method 5). Yield $68 \%$. The product gradually became dark in colour, more rapidly on exposure to air than in closed vessels.

$\alpha$-Iodoethyl acetate was prepared from acetyl iodide and paraldehyde (Lachema) (method 5). The reaction was vigorous; paraldehyde was therefore added slowly. Yield $43 \%$. The product could not be obtained wholly colourless, and it darkened in air so rapidly that an accurate determination of the refractive index was not possible.

Dichloromethyl formate was prepared from phenyl(bromodichloromethyl)mercury and formic acid (E. Merck AG, Guaranteed Reagent) (method 8). The ester contained variable amounts of benzene as found by gas-liquid chromatography and from infrared spectra; no analytical data are therefore given. The ester was found to be unstable as has been observed earlier by Grignard et al. ${ }^{45}$ who found that dichloromethyl formate is completely transformed during chlorination into the chloroformate derivatives, and by Delépine, ${ }^{46}$ who assumed that the rapid decomposition of some chlorination products of methyl formate was caused by the hydrolysis of dichloromethyl formate catalysed by traces of water through the reactions

$$
\begin{aligned}
& \mathrm{HCOOCHCl}+2 \mathrm{H}_{2} \mathrm{O}=\mathrm{HCOOCOH}+2 \mathrm{HCl} \\
& -\mathrm{HCOOCOH}=2 \mathrm{CO}+\mathrm{H}_{2} \mathrm{O}
\end{aligned}
$$

The proposed decomposition of dichloromethyl formate via formic anhydride was inves tigated by adding a sample of the ester with a small amount of water (ca. 1 mole \%) to a vessel equipped with a manometer. The ester was, indeed, found to decompose during the course of several months at an approximately constant rate. When about $0.1 \mathrm{mmole}$ of water was added to 10 mmoles of the ester, the rate of decomposition at room temperature was about $0.05 \mathrm{mmole}$ per day. On the other hand, the ester hydrolysed in homogeneous aqueous solutions or heterogeneous mixtures containing water like other dichloromethyl esters ${ }^{47}$ according to the equation

$$
\mathrm{HCOOCHCl}_{2}+\mathrm{H}_{2} \mathrm{O}=\mathrm{HCOOH}+\mathrm{CO}+2 \mathrm{HCl}
$$

Acta Chem. Scand. 20 (1966) No. 5 
as indicated by the ratio 3:2 of the total equivalents of formed acids to those of the chloride ion and by the evolution of carbon monoxide.

Dichloromethyl chloroacetate ${ }^{27}$ was prepared from phenyl(bromodichloromethyl)mercury and chloroacetic acid (method 8 ). Yield $86 \%$.

Dichloromethyl trichloroacetate was prepared by chlorinating methyl trichloroacetate (method 1). After careful distillation, the purest fractions were treated with the calculated amount of water in acetone for the hydrolysis of the trichloromethyl ester which had not been removed by distillation. After redistillation with a high refluxing ratio, pure dichloromethyl trichloroacetate was obtained. Anschütz and Emery ${ }^{48}$ and Iliceto ${ }^{36}$ mention the occurrence of the ester among the chlorination products of methyl trichloroacetate, but it does not seem to have been prepared in the pure state.

Trichloromethyl trichloroacetate ${ }^{\mathbf{4 8 , 2 9}}$ was prepared by chlorinating cold methyl trichloroacetate (method 1). After careful distillation, the ester was crystallised three times from hexane; m.p. $33.5^{\circ} \mathrm{C}$ (Anschütz and Emery ${ }^{48}$ give the value $34^{\circ} \mathrm{C}$ ).

Acknowledgments. The authors wish to thank the National Research Council for Sciences (Valtion Luonnontieteellinen Toimikunta) for financial aid and Mr. Nils J. Cleve, $\mathrm{Ph}$. M., for help with the analytical work.

\section{REFERENCES}

1. Euranto, E. Ann. Univ. Turku., Ser. A I 1959 No. 31, pp. 11-17.

2. Euranto, E. and Kujanpää, T. Acta Chem. Scand. 15 (1961) 1209.

3. Euranto, E. K. and Leppänen, O. Acta Chem. Scand. 17 (1963) 2765.

4. Euranto, E. Suomen Kemistilehti B 33 (1960) 41.

5. Euranto, E. Ann. Univ. Turku., Ser. A I 1960 No. 42.

6. Euranto, E. Suomen Kemistilehti B 35 (1962) 18.

7. Euranto, E. K. and Euranto, R. A. Suomen Kemistilehti B 35 (1962) 96.

8. Cleve, N. J. and Euranto, E. K. Suomen Kemistilehti B 37 (1964) 126.

9. Euranto, E. K. and Yrjänä, T. Suomen Kemistilehti B 38 (1965) 215.

10. Laato, H. and Himanka, E. Suomen Kemistilehti B 37 (1964) 85.

11. Shlichta, P., Inman, J. K. and Lucas, H. J. J. Am. Chem. Soc. 77 (1955) 3784.

12. Bavin, P. M. G. Can. J. Chem. 42 (1964) 704.

13. Bruylants, A., Tits, M. and Dauby, R. Bull. Soc. Chim. Belges 58 (1949) 310; Bruylants A., Tits, M., Dieu, C. and Gauthier, R. Ibid. 61 (1952) 366.

14. Singh, H. and Tedder, J. M. J. Chem. Soc. 19644737.

15. Brace, N. O. and McCormack, W. B. J. Org. Chem. 26 (1961) 5091.

16. British Patent 325,115 (1929); Chem. Abstr. 24 (1930) 3800.

17. German Patent 313,696 (1919); Chem. Zentr. 90 (1919) IV 664.

18. Kirrmann, A. Bull. Soc. Chim. France [5] 4 (1937) 502.

19. Kharasch, M. S., Reinmuth, O. and Urry, W. H. J. Am. Chem. Soc. 69 (1947) 1105.

20. Descudé, M. Compt. Rend. 132 (1901) 1567.

21. Imperial Chemical Industries Ltd. British Patent 837,486 (1960).

22. Descudé, M. Bull. Soc. Chim. France [3] 27 (1902) 867.

23. Pishnamazzade, B. F. and Gulieva, Sh. D. Dokl. Akad. Nauk Azerb. SSR 12 (1956) 895; Pishnamazzade, B. F., Gasanova, Sh. D. and Kerimova, R. M. Ibid. 19 (1963) No.11, 23.

24. Arundale, E. U. S. Patent 2,607,800 (1952); Chem. Abstr. 48 (1954) $7052 b$.

25. Reppe, W. et al. Ann. 596 (1955) 1, pp. 89 and 91.

26. German Patent 1,081,450 (1960); Chem. Abstr. 55 (1961) $14479 \mathrm{e}$.

27. Seyferth, D., Mui. J. Y.-P. and Todd, L. J. J. Am. Chem. Soc. 86 (1964) 2961; Seyferth, D., Burlitch, J. M., Minasz. R. J., Mui, J. Y.-P., Simmons, H. D., Jr., Treiber, A. J. H. and Dowd, S. R. J. Am. Chem. Soc. 87 (1965) 4259.

28. Reutov, O. A. and Lovtsova, A. N. Dokl. Akad. Nauk SSSR 139 (1961) 622; Seyferth, D. and Burlitch, J. M. J. Am. Chem. Soc. 84 (1962) 1757; J. Organometal. Chem. 4 (1965) 127.

29. Elbs, K. and Kratz, K. J. prakt. Chem. [2] 55 (1897) 502.

30. Kaufler, F. and Herzog, C. Ber. 42 (1909) 3858.

Acta Chem. Scand. 20 (1966) No. 5 
31. Laato, H. Ann. Univ. Turku., Ser. $A I$ (1965) Nos. 82 and 86.

32. Yarovenko, N. N., Raksha, M. A., Shemanina, V. N. and Vasil'eva, A. S. Zh. Obshch. Khim. 27 (1957) 2246.

33. Talbott, R. L. J. Org. Chem. 30 (1965) 1429.

34. Mitsch, R. A. Unpublished work. ${ }^{33}$

35. Descudé, M. Compt. Rend. 136 (1903) 1565.

36. Iliceto, A. Ann. Chim. (Rome) 43 (1953) 638.

37. Vogel, A. I. J. Chem. Soc. 19481833.

38. Chancel, P. Bull. Soc. Chim. France [5] 18 (1951) 228.

39. Brown, H. C. and Ash, A. B. J. Am. Chem. Soc. 77 (1955) 4019.

40. Späth, E. and Schmid, H. Ber. B 73 (1940) 243.

41. Ulich, L. H. and Adams, R. J. Am. Chem. Soc. 43 (1921) 660.

42. Gayler, C. W. and Waddle, H. M. J. Am. Chem. Soc. 63 (1941) 3358.

43. Euranto, E. K. and Moisio, A.-L. Suomen Kemistilehti B 37 (1964) 92.

44. Renshaw, R. R. and Ware, J. C. J. Am. Chem. Soc. 47 (1925) 2989.

45. Grignard, V., Rivat, G. and Urbain, E. Compt. Rend. 169 (1919) 1074; Ann. Chim. (Paris) 9 (1920) 229.

46. Delépine, M. Bull. Soc. Chim. France [4] 27 (1920) 39.

47. Laato, H. Suomen Kemistilehti B 37 (1964) 11.

48. Anschütz, R. and Emery, W. O. Ann. 273 (1893) 56.

Received February 7, 1966. 